\title{
Produção de biodiesel por transesterificação enzimática in situ em solvente orgânico a partir da biomassa de Desmodesmus sp.
}

\author{
Danielle Kochenborger John ${ }^{1}$ \\ Martiele Bizarro da Silva ${ }^{2}$ \\ Michele Hoeltz ${ }^{3}$ \\ Adilson Ben da Costa ${ }^{4}$ \\ Rosana de Cássia de Souza Schneider ${ }^{5}$
}

\section{RESUMO}

Um dos materiais alternativos mais investigados atualmente para a produção de biodiesel são as microalgas. O presente trabalho teve como objetivo a avaliação do método de transesterificação enzimática in situ da microalga Desmodesmus sp. em solvente orgânico, acompanhando a reação através do monitoramento on-line com sonda de infravermelho e cromatografia gasosa. Dois ensaios foram realizados em condições distintas para efeito comparativo. Baseado nos resultados obtidos por espectroscopia e cromatografia, bem como nas propriedades calculadas a partir do perfil cromatográfico, pode-se concluir que é possível à produção de biodiesel in situ a partir de biomassa de microalgas, reduzindo a etapa de extração de óleo.

Palavras-chave: Microalga. Desmodesmus sp. Biodiesel. Transesterificação in situ. Espectroscopia de infravermelho. Cromatografia gasosa.

\begin{abstract}
One of the most investigated alternative materials for the production of biodiesel is microalgae. The present work had the objective of evaluating the in situ enzymatic transesterification method using the microalga Desmodesmus sp. in organic solvent, following the reaction through online monitoring with infrared spectroscopy and gas chromatography. Two experiments were carried out under different conditions for comparative effect. Based on the results obtained by spectroscopy and chromatography, as well as the properties calculated from the chromatographic profile, it can be concluded that it is possible to produce in situ biodiesel from microalgae biomass, eliminating the oil extraction step.
\end{abstract}

Keywords: Microalgae. Desmodesmus sp. Biodiesel. in situ Transesterification. Infrared spectroscopy. Gas chromatography.

\footnotetext{
${ }^{1}$ Aluna do Curso de Química Industrial da Universidade de Santa Cruz do Sul.

${ }^{2}$ Aluna do Curso de Ciências Biológicas - Bacharelado da Universidade de Santa Cruz do Sul.

${ }^{3}$ Professora do Departamento de Biologia e Farmácia e Programa de Pós-Graduação em Tecnologia Ambiental na Universidade de Santa Cruz do Sul.

${ }^{4}$ Professor do Departamento de Biologia e Farmácia e Programa de Pós-graduação em Sistemas e Processos Industriais na Universidade de Santa Cruz do Sul.

${ }^{5}$ Professora do Departamento de Química e Física e Programa de Pós-Graduação em Tecnologia Ambiental na Universidade de Santa Cruz do Sul. <rosana@unisc.br>
} 


\section{INTRODUÇÃO}

Microalgas são micro-organismos fotossintéticos simples e de grande importância no equilíbrio de sistemas aquáticos. Podem se adaptar a diversos ambientes e suportar mudanças de temperatura e composição do meio, incidência de radiações e anaerobiose. As microalgas, pela extensa variedade de espécies e propriedades intrínsecas, demonstram grande potencial para exploração comercial. Dentre os principais componentes de suas células, destaca-se seu teor de lipídios, que é matéria prima para a produção de biodiesel (CAMPANELLA et al., 2001, MALLICK, 2002; CHISTI, 2007; AMARO et al., 2012).

A preocupação com o aumento de lançamento de $\mathrm{CO}_{2}$ e outros poluentes na atmosfera é um assunto premente. Conforme Singh et al. (2014) o crescimento de $2 \%$ ao ano de energia para transporte pode conduzir a $80 \%$ mais emissões de gases de efeito estufa em comparação aos níveis de 2002.

Novas estratégias para a substituição das energias fósseis necessitam ser constantemente desenvolvidas. Fontes renováveis como energia solar, eólica, geotérmica e a partir de biomassa vegetal têm a capacidade de prover alternativas para uma energia mais limpa (SINGH et al., 2014). Segundo Beer et al. (2011), o consumo de combustíveis renováveis tem impacto ambiental reconhecidamente menor. O óleo diesel, um combustível fóssil responsável por boa parte da emissão dos gases responsáveis pelo efeito estufa, pode ser em parte substituído pelo uso de biodiesel de diversas culturas, entre elas as microalgas.

O biodiesel consiste na conversão de triacilgliceróis (TAG) por transesterificação, onde TAG reagem com álcoois, na presença de um catalisador, para a formação de ésteres alquílicos de ácidos graxos (EMAG) (HOEKMAN et al., 2012), como ilustrado na Figura 1. As vantagens do biodiesel sobre o diesel derivado do petróleo incluem uso de matéria prima renovável, aumento da capacidade de lubrificação, biodegradabilidade, menor toxicidade e redução na emissão de monóxido de carbono (CO) (LEE et al., 2010). 


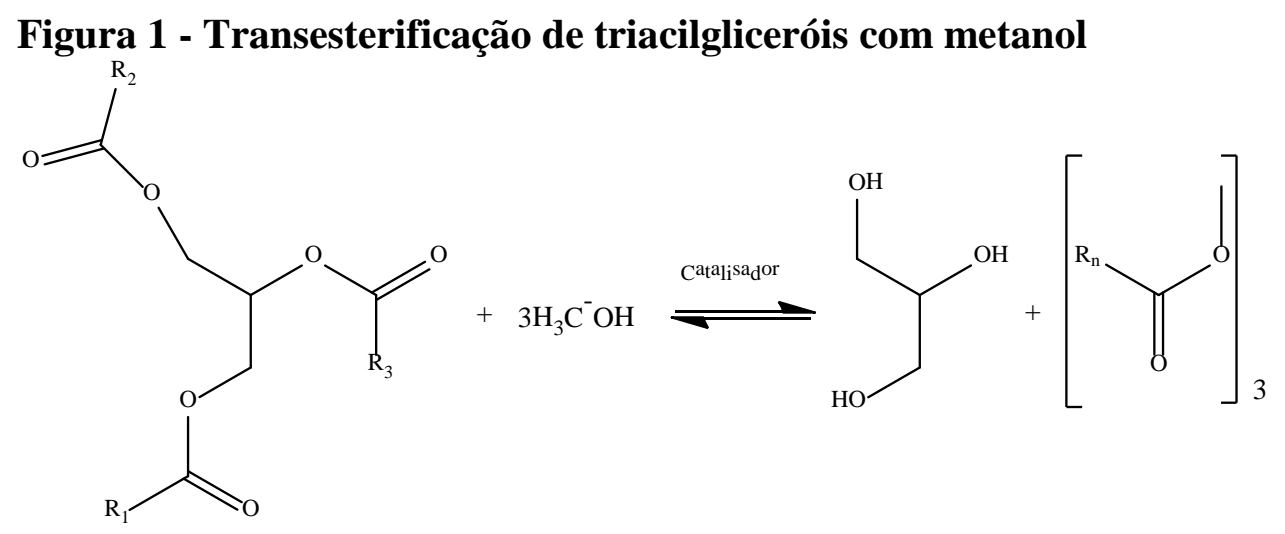

Fonte: Hoekman et al., 2012

Os métodos tradicionais de produção de biodiesel partem da conversão de óleos vegetais de plantas superiores oleaginosas. Para o emprego de microalgas como matéria-prima, a produção de biodiesel requer duas etapas para a obtenção dos ésteres metílicos: (1) extração de lipídios e (2) transesterificação. Como alternativa, a etapa de extração pode ser eliminada realizando a reação diretamente no conteúdo lipídico contido na biomassa, em um processo conhecido como transesterificação in situ.

Metodologias que fazem uso de técnicas de transesterificação in situ se tornam alternativas para a eliminação das limitações e perdas sofridas pelos processos convencionais de extração e conversão, integrando estas etapas e reduzindo custos (DONG et al., 2013). Velasquez-Orta et al. (2012) alcançaram 77,6\% de conversão em biodiesel com catalisador alcalino partindo de biomassa de Chlorella vulgaris como fonte de lipídios, combinando as etapas de extração e transesterificação.

A combinação da transesterificação in situ a um catalisador enzimático é outra maneira de aprimorar a produção de biodiesel. A utilização de enzimas é capaz de atingir alta conversão, com rendimentos entre 86-96\%. Catalisadores enzimáticos são seletivos, específicos e ambientalmente menos impactantes, acarretando na diminuição da formação de coprodutos durante a reação e na sua reutilização (GO et al., 2016). Guldhe et al. (2016) obtiveram conversão à EMAG dos lipídios da microalga Scenedesmus obliquus de 90,82\%, utilizando lipase imobilizada do Aspergillus niger e adição de metanol em etapas. Esta técnica possui vários pontos fortes em relação ao uso de outros catalisadores, principalmente a baixa quantidade de resíduos e custo de energia, pois pode ser realizada em temperaturas ambientes. 
O monitoramento da conversão de TAG em biodiesel nas reações in situ é um desafio, uma vez que a ruptura celular pode liberar mais compostos, além de óleo que será convertido em éster. Os principais métodos para acompanhamentos são cromatografia gasosa (CG) (KEERA et al., 2011), análises termogravimétricas (TGA) (SOUSA et al., 2013) e cromatografia líquida de alta eficiência (CLAE) (LI et al., 2001). Os processos instrumentais de análise de biodiesel são lentos, com preços elevados, exigindo operadores altamente qualificados e não são facilmente adaptados ao monitoramento on-line (MUELLER et al., 2013). O desenvolvimento da espectroscopia por infravermelho tem proporcionado a obtenção informações qualitativas e quantitativas sobre a amostra de interesse de uma maneira mais avançada e menos destrutiva (ZHANG, 2012), ou mesmo direto no meio reacional sem alterar os resultados e identificando modificações durante toda a reação (OLIVEIRA et al., 2017).

Neste contexto, o presente trabalho teve como objetivo avaliar a produção do biodiesel a partir da biomassa da microalga Desmodesmus sp., através do método de transesterificação enzimática in situ utilizando solvente orgânico como meio reacional, acompanhando a biotransformação através de espectrometria no infravermelho e cromatografia gasosa.

\section{MATERIAIS E MÉTODOS}

Os experimentos foram realizados visando à comparação de dois métodos de produção de biodiesel de microalgas, como mostra a Figura 2. 
Figura 2 - Diagrama comparativo entre as principais etapas nos processos de produção de biodiesel convencional e in situ

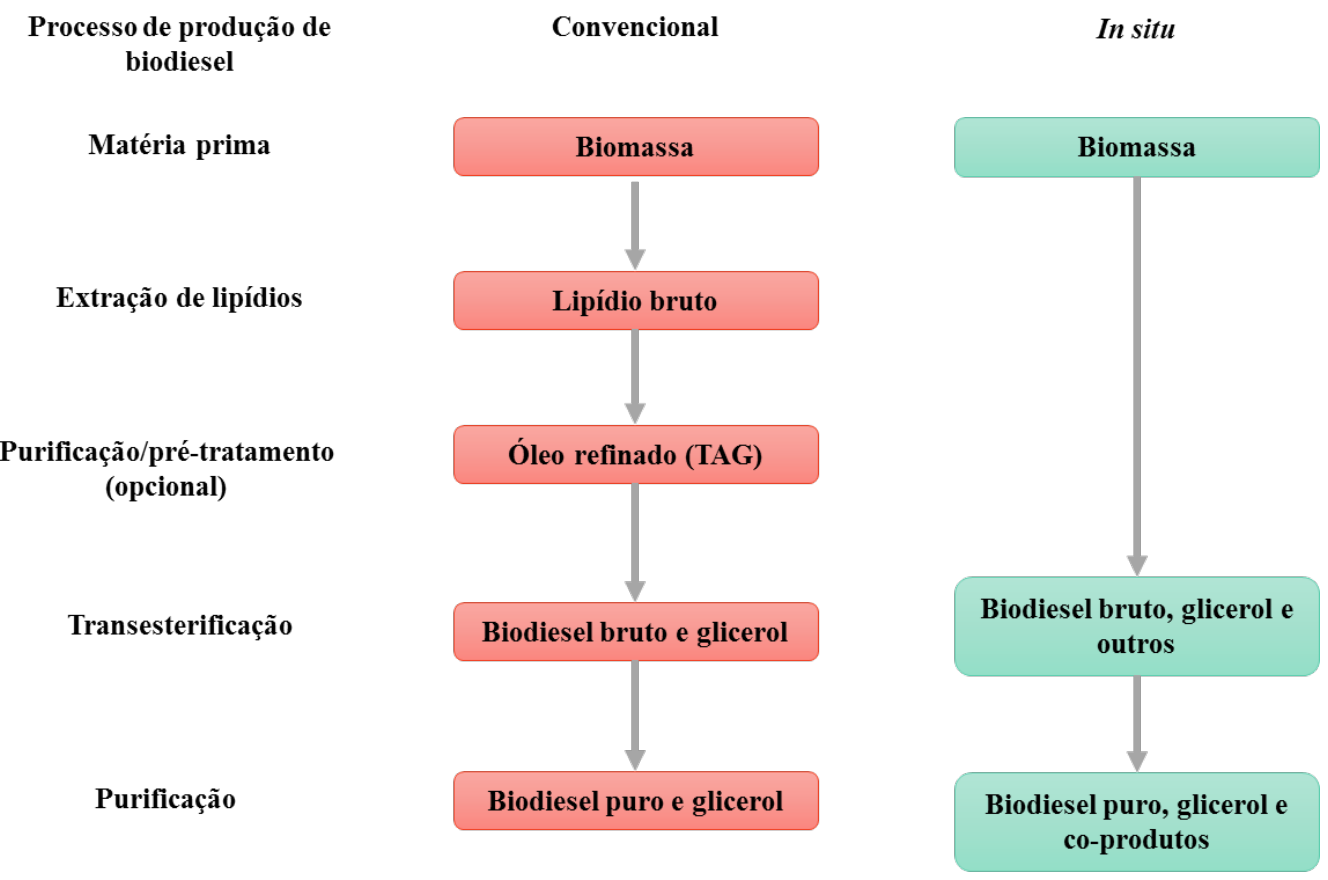

Fonte: O Autor

\subsection{Cultivo da microalga}

A cepa da microalga Desmodesmus sp. foi obtida no Laboratório de Ecotoxicologia da Universidade de Santa Cruz do Sul. Inicialmente foi aclimatada em meio contendo nitrogênio, fósforo e potássio (N:P:K, 18:6:18 m/m) a $3 \mathrm{~g} \mathrm{~L}^{-1}$. Este pré-inóculo foi transferido para um fotobiorreator tubular tipo coluna de bolhas (GRESSLER et al., 2014), onde repiques semanais foram realizados para manutenção da cultura. O sistema foi mantido à temperatura constante de $25^{\circ} \mathrm{C}$ e iluminação contínua com lâmpadas de $20 \mathrm{~W}$. Para o acompanhamento do crescimento da microalga foi utilizado o método de Chiu et al. (2008). A densidade celular foi obtida através da construção de uma curva analítica, medindo sua absorbância em espectrofotômetro UV-vis Q798U (Quimis ${ }^{\circledR}$ ) a $682 \mathrm{~nm}$. A amostra foi diluída para que se obtivesse valores de absorbância entre 0,1 e 1,0. O monitoramento foi realizado para observação do crescimento e manutenção do cultivo. 


\subsection{Separação da biomassa e rendimento de massa seca}

A biomassa foi separada por centrifugação, utilizando centrífuga (6-16 KS Sigma ${ }^{\circledR}$ ), em 3600 rpm, por 20 minutos, à $20^{\circ} \mathrm{C}$. Posteriormente, a biomassa colhida foi congelada, liofilizada (liofilizador K120 Liotop $^{\circledR}$ ) e armazenada.

\subsection{Determinação de teor de clorofila e carotenoides}

As concentrações de clorofila e carotenoides foram determinadas pelo método de Lichtenthaler (1987), utilizando acetona 80\% (v/v) para a extração e leitura da absorbância da solução em diferentes comprimentos de onda em espectrofotômetro UV/visível (Q798U Quimis $^{\circledR}$ ). A determinação dos pigmentos foi realizada a partir das equações 1 a 3.

$C_{a}=12,25 A_{663}-2,79 A_{647}$

$C_{b}=21,50 A_{647}-2,79 A_{663}$

Carotenoides $=1000 A_{470}+1,82 C_{a}+\frac{85,02 C_{b}}{198}$

Onde A é a absorbância medida nos respectivos comprimentos de onda, $\mathrm{C}_{\mathrm{a}}$ é o teor de Clorofila a e $C_{b}$ é o teor de Clorofila b. Os valores encontrados em $\mu g \mathrm{~mL}^{-1}$ de extrato, foram convertidos à $\mu \mathrm{g} \mathrm{g}^{-1}$ de biomassa seca.

\subsection{Determinação de lipídios e extração de óleo da biomassa}

Para a quantificação de lipídios foi utilizado o método adaptado de Bligh eDyer (1959). Foram adicionados $6 \mathrm{~mL}$ da mistura clorofórmio e metanol (1:2 v/v) à $100 \mathrm{mg}$ de biomassa liofilizada. A amostra foi sonicada (ECO-SONICS Q 1.8/25 ${ }^{\circledR}$ ) por 30 minutos e levada ao freezer por 4 horas. Após, foi novamente sonicada por 30 minutos e centrifugada por 20 minutos (centrífuga refrigerada 6-16 KS Sigma ${ }^{\circledR}$ ) à $2000 \mathrm{rpm}$ e $15^{\circ} \mathrm{C}$. O sobrenadante foi recuperado em novo frasco, ao qual foram adicionados $4 \mathrm{~mL}$ de água deionizada e $2 \mathrm{~mL}$ de clorofórmio e levada a centrifugação até sedimentação. A fase orgânica contendo os lipídios foi transferida ao frasco pré-pesado. O solvente foi evaporado sob fluxo de nitrogênio.

\subsection{Derivatização do óleo extraído da biomassa}

Em um balão de fundo redondo, ao óleo extraído pelo método de extração de lipídios adaptado de Bligh eDyer (1959), foram adicionados 6,0 $\mathrm{mL}$ de uma solução metanólica de $\mathrm{NaOH} 2 \%$ e aqueceu-se sob refluxo por 20 minutos. Adicionou-se, gota a gota, $7 \mathrm{~mL}$ de solução 
$\mathrm{BF}_{3} /$ Metanol. Após 4 minutos, $5 \mathrm{~mL}$ de heptano foram acrescentados e deixou-se a amostra em refluxo por 2 minutos. Posteriormente, o balão foi retirado do aquecimento e resfriado a temperatura ambiente. Uma solução saturada de $\mathrm{NaCl}$ foi acrescida e agitada suavemente até a observação de duas fases. Transferiu-se para um tubo de ensaio e a fase superior foi separada para a adição de $\mathrm{Na}_{2} \mathrm{SO}_{4}$ anidro. A parte líquida foi retirada e armazenada para posterior análise cromatográfica.

\subsection{Composição centesimal}

Os teores de umidade, cinzas, proteínas, fibras e carboidratos foram determinados na biomassa inicial seguindo os procedimentos elaborados pelos Métodos Oficiais de Análise AOAC (Association of Official Analytical Chemists, 1990).

\subsection{Transesterificação enzimática in situ}

As reações foram realizadas partindo da biomassa liofilizada e do óleo extraído, utilizado como referência. O óleo foi extraído da biomassa a partir do método Bligh Dyer (1959) modificado para extração de óleo a partir de uma massa maior de biomassa, com 4 gramas. Lipase imobilizada em resina acrílica Novozym $^{\circledR} 435$ foi usada como catalisador, na concentração de $10 \%$ em relação os respectivos pesos de biomassa e óleo. Os ensaios foram acompanhados por Espectroscopia de Infravermelho com Transformada de Fourier com Reflectância Total Atenuada (ATR - FTIR) através da sonda ReactIR 15 (Mettler Toledo ${ }^{\circledR}$ ).

\subsection{Métodos de acompanhamento da transesterificação}

\subsubsection{Espectroscopia no Infravermelho}

Conforme descrito no item 2.7, as reações foram monitoradas com a sonda ReactIR 15 (Mettler Toledo ${ }^{\circledR}$ ) também foram obtidos os espectros da biomassa, dos padrões dos solventes hexano, metanol, água, do óleo extraído e do óleo derivatizado. O espectro da biomassa foi obtido através da elaboração de um filme sobre a superfície de leitura da sonda de infravermelho.

A faixa espectral coletada foi de 650 a $2500 \mathrm{~cm}^{-1}$, com resolução de $8 \mathrm{~cm}^{-1}$ e 256 varreduras e as leituras foram realizadas automaticamente a cada 5 minutos de reação, por 48 horas. 


\subsubsection{Cromatografia gasosa}

A análise quantitativa foi realizada por cromatografia gasosa com detecção por ionização em chama (CG-DIC) em um equipamento Shimadzu ${ }^{\circledR}$ QP 2010 Plus equipado com Injetor Automático AOC 20i, sendo as amostras separadas em uma coluna capilar Zbwax (30 m x 0,25 mm x 0,25 $\mu \mathrm{m}$ ). O hélio (White Martins Gases Industriais, Canoas, Brasil, 99,999\%) foi utilizado como gás de arraste e a sua taxa de fluxo foi de $1 \mathrm{~mL} \mathrm{~min}^{-1}$. A programação de temperatura do forno foi $70^{\circ} \mathrm{C}, 4^{\circ} \mathrm{C} \min ^{-1}$, até $240{ }^{\circ} \mathrm{C}, 5^{\circ} \mathrm{C} \mathrm{min}{ }^{-1}$, até $250^{\circ} \mathrm{C}$, totalizando 49,5 minutos de análise. Temperatura do detector $300{ }^{\circ} \mathrm{C}$, temperatura do injetor $250{ }^{\circ} \mathrm{C}$, operado no modo "split" 1:1 e volume de injeção de $1 \mu \mathrm{L}$. A identificação dos compostos presentes foi efetuada pela comparação dos tempos de retenção com padrões comerciais de ésteres metílicos (Supelco Analitycal ${ }^{\circledR}$ - Bellefonte, Pennsylvania, USA).

Após a análise cromatográfica, as propriedades do biodiesel foram estimadas utilizando “Biodiesel Analyzer ${ }^{\odot}$ Ver. 1.1” (disponível em http://www.brteam.ir/biodieselanalyzer).

\section{RESULTADOS E DISCUSSÃO}

\subsection{Produção de biomassa}

\subsubsection{Rendimento da biomassa liofilizada}

Após centrifugada e liofilizada, o rendimento de biomassa seca da microalga Desmodesmus sp. foi de 508,96 $\pm 105,97 \mathrm{mg} \mathrm{L}^{-1}$. O valor obtido foi superior ao encontrado por Gressler et al. (2014) que, utilizando efluente da Estação de Tratamento de Esgoto da Universidade de Santa Cruz do Sul (ETE-UNISC) como meio de cultivo, obteve 234,00 $\mathrm{mg} \mathrm{L}^{-1} \pm$ $91,89 \mathrm{mg} \mathrm{L}^{-1}$ para a mesma microalga. O mesmo estudo observou que o aporte de $\mathrm{CO}_{2}$, nos parâmetros estabelecidos, pode aumentar a produção de biomassa microalgal para 1277,44 \pm 1053,04 $\mathrm{mg} \mathrm{L}^{-1}$. Incrementos no meio de cultivo no qual a microalga foi cultivada podem melhorar seu rendimento em biomassa e conteúdo lipídico.

\subsubsection{Caracterização da biomassa}

Produção de lipídios é um dos principais focos para o uso de microalgas como alternativa para a produção de biocombustíveis. A fração lipídica média da biomassa foi de 8,21 \pm 0,62\% para o cultivo em N:P:K, como mostra a Tabela 1. Microalgas podem apresentar entre 1\% e 70\% de conteúdo lipídico, embora a maioria das espécies não ultrapasse de 40\%, e sua composição 
varie de acordo com a espécie, constituição genética e meio de cultivo (ZMORA; RICHMOND, 2004; VIJAYARAGHAVAN; HEMANATHAN, 2009).

Tabela 1 - Composição centesimal da biomassa seca da microalga Desmodesmus sp. cultivada em fotobiorreator com N:P:K como meio de cultivo

\begin{tabular}{cc}
\hline Composição centesimal & Valor em biomassa seca de Desmodesmus sp. (\%) \\
\hline Umidade & $1,74 \pm 0,04$ \\
Proteínas & $33,91 \pm 0,39$ \\
Cinzas & $17,46 \pm 2,17$ \\
Lipídios & $8,21 \pm 0,62$ \\
Fibras & $21,40 \pm 4,20$ \\
Carboidratos & $17,27 \pm 2,60$ \\
\hline
\end{tabular}

Fonte: O Autor.

A biomassa apresentou 17,27 $\pm 2,60 \%$ de carboidratos, que são constituintes da parede celular da microalga, como celulose, pectina ou polissacarídeos. Estes componentes variam de acordo com a espécie e também são acumulados como reserva de energia nos plastídios, na forma de amido, podendo chegar até $60 \%$ em algumas espécies. O acúmulo de carboidratos aumenta devido à limitação de nutrientes no meio. A espécie Chlorella vulgaris é considerada uma das principais fontes de carboidratos, principalmente de açúcares fermentescíveis, responsáveis pela produção de bioetanol (CHEN et al., 2013). O teor de fibras (21,40 \pm 4,20 \%) pode indicar nível de celulose, uma fibra insolúvel que, juntamente com os carboidratos, pode ser convertido em açúcares de cadeia menores através de hidrólise (KIM; KIM, 2013). Outros componentes como as proteínas, podem ser utilizados para nutrição humana e animal, sendo que em algumas espécies, como a Spirulina máxima, podem chegar a 70\% do conteúdo da biomassa seca e não costumam ser utilizadas para a produção de biocombustíveis (HARUN et al., 2010). Já as cinzas indicam o total de componentes inorgânicos presentes na biomassa, ficando acima do encontrado na literatura, onde, para a espécie Desmodesmus communis, em diferentes meios de cultivo, obteve-se valores entre 3 e 13,3\% (SAMORÌ et al., 2013).

A composição total das microalgas pode também conter ceras, esteróis, terpenos, quinonas e pigmentos (JUNEJA et al., 2013). Dentre os principais componentes corantes estão as clorofilas, que possuem atividade quelante, podendo ser utilizadas na área farmacêutica, pois aumentam o nível de crescimento celular, acelerando a cicatrização, além de ser utilizada na indústria alimentícia como corante, devido a intensidade de sua coloração verde (HARUN et al., 2010). 
Os carotenoides são substâncias encontradas em diversas plantas superiores e algas, assim como em organismos não fotossintéticos e são responsáveis pela coloração avermelhada. Nas algas, os mesmos possuem atividade essencial na realização de fotossíntese, juntamente com a clorofila, mantendo a estrutura e funções de complexos fotossintéticos. Possuem ação antioxidante, protegendo estes micro-organismos contra o estresse oxidativo (DEL CAMPO et al., 2007). A Tabela 2 apresenta os teores de clorofilas e carotenoides da biomassa da microalga Desmodesmus sp.

Devido à procura por corantes naturais nas indústrias alimentícia e cosmética, estudos propõem métodos de aumentar a produtividade de determinados pigmentos através da modificação de meios de cultivo das microalgas produtoras de corantes. Os valores encontrados de clorofila totais foram considerados abaixo dos encontrados em outras espécies de algas verdes, como a Chlorella pyrenoidosa, que possuem percentual de clorofila entre 0,01 e 6,00\% (Hodaifa et al., 2008). Hu et al. (2013), utilizando um meio altamente salino e iluminação para a microalga Coelastrella sp. F50, conseguiram diminuir o tempo de produção de carotenoides de um mês para doze dias. Utilizando a microalga Spirulina platensis em um meio com ureia como fonte de nitrogênio, foi possível aumentar a quantidade de clorofila produzida, na busca de fontes não-vegetais deste corante (DE OLIVEIRA RANGEL-YAGUI et al., 2004).

Tabela 2 - Teor de Carotenoides e Clorofilas a, b e totais da microalga Desmodesmus sp. em $\mu \mathrm{g}^{-1}$ de biomassa seca

\begin{tabular}{cc}
\hline Pigmento & Concentração $\left(\boldsymbol{\mu g} \boldsymbol{g}^{-1}\right)$ \\
\hline Clorofila $a$ & $220,67 \pm 54,27$ \\
Clorofila $b$ & $84,40 \pm 13,78$ \\
Clorofilas totais & $297,74 \pm 69,69$ \\
Carotenoides & $47,36 \pm 13,31$ \\
\hline
\end{tabular}

Fonte: O Autor

\subsection{Transesterificação Enzimática}

\subsubsection{Espectroscopia no Infravermelho}

As diferentes reações de transesterificação foram monitoradas em tempo real coletando espectros no infravermelho por ATR-FTIR. Para cada reação, foi obtido um conjunto de 576 espectros, que puderam ser plotados, formando um gráfico tridimensional (Figura 3), sendo 
monitorada a mudança de intensidade na região de 1300 - $1100 \mathrm{~cm}^{-1}$, correspondente ao estiramento da ligação C-O nos ésteres, e a região de 1750 - $1725 \mathrm{~cm}^{-1}$ característica tanto dos ésteres metílicos quanto dos TAG. Outras regiões também foram observadas para a caracterização, conforme a Tabela 3. As análises cromatográficas foram realizadas nas amostras de forma que os ésteres metílicos fossem quantificados, através da detecção por ionização em chama (CG-DIC).

O ensaio in situ apresentou aumento de intensidade das bandas, comumente atribuídas aos ésteres depois de 15h de reação. Após 34h, puderam ser observadas variações de intensidade nas bandas $1656 \mathrm{~cm}^{-1}$ e $1134 \mathrm{~cm}^{-1}$. Estas mudanças sugerem o consumo do componente anteriormente liberado no meio. As mesmas bandas sofrem aumento de absorção de energia na região do IV e, por esta região também indicar presença de ésteres, mostrou a biotransformação dos ácidos graxos na transesterificação.

Acrescenta-se que o emprego do monitoramento da reação por infravermelho permitiu identificar que a reação alcançou o equilíbrio químico. Pode-se acompanhar a mudança de intensidade da banda de estiramento C-O, atribuída aos ésteres na região de 1300-1100 cm ${ }^{-1}$ durante as 10 primeiras horas de reação, observado na Figura 4. Verificou-se, portanto que há uma mudança tanto no ensaio in situ, utilizando a biomassa, que pode ser relacionada à liberação do conteúdo lipídico ao meio reacional, quanto no ensaio utilizando o óleo extraído, indicando o consumo de material no meio e sua biotransformação.

Figura 3 - Conjunto de espectros coletados durante o processo de transesterificação enzimática a partir da biomassa microalgal (a) e do óleo previamente extraído (b)
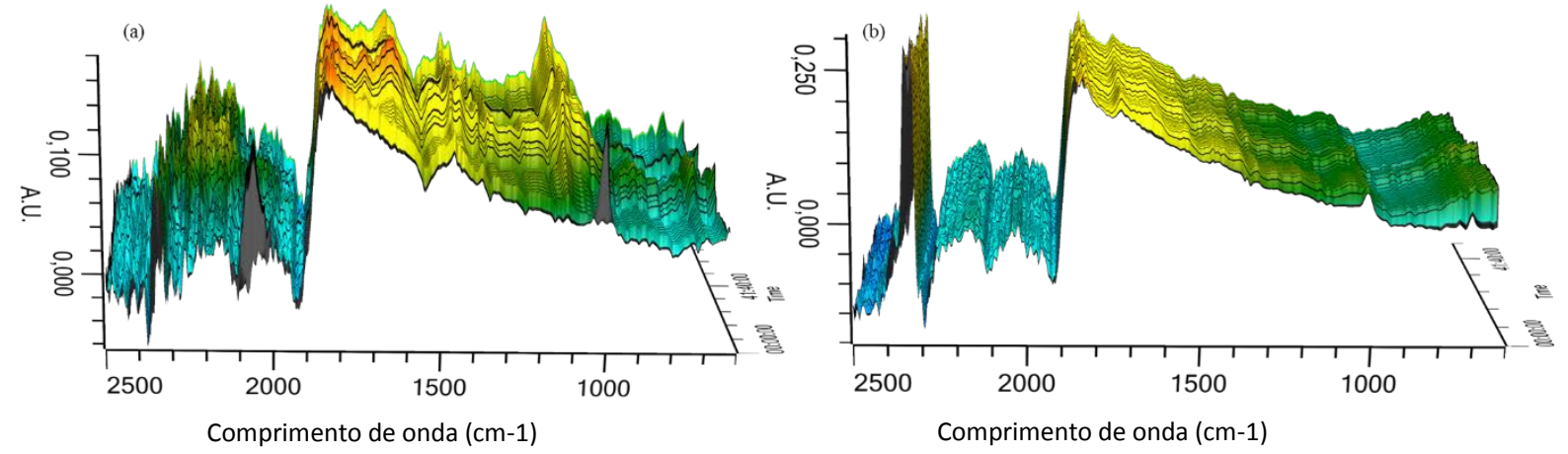

Fonte: O Autor. 
Tabela 3 - Principais bandas de absorção de microalgas no IV

\begin{tabular}{|c|c|c|c|}
\hline $\begin{array}{c}\text { Número de } \\
\text { onda } \\
\left(\mathrm{cm}^{-1}\right)\end{array}$ & Grupo funcional & $\begin{array}{l}\text { Classe do composto } \\
\text { associado }\end{array}$ & Referência \\
\hline$\sim 1750-1725$ & estiramento $\mathrm{C}=\mathrm{O}$ & ésteres, lipídios & \\
\hline$\sim 1655$ & estiramento $\mathrm{C}=\mathrm{O}$ & proteínas & \\
\hline$\sim 1545$ & $\begin{array}{l}\text { deformação N-H, } \\
\text { estiramento C-N }\end{array}$ & proteínas & (Mayers et al., 2013) \\
\hline$\sim 1390$ & $\begin{array}{l}\text { deformação } \mathrm{CH}_{2} \text {, } \\
\text { deformação } \mathrm{CH}_{3}\end{array}$ & grupos metil e metileno & \\
\hline $1300-1100$ & estiramento C-O & ésteres & $\begin{array}{c}\text { (de Souza e Cajaiba da } \\
\text { Silva, 2012) }\end{array}$ \\
\hline$\sim 1240$ & estiramento $\mathrm{P}=\mathrm{O}$ & $\begin{array}{l}\text { fosfodiésteres e } \\
\text { fosfolipídios }\end{array}$ & (Mayers et al., 2013) \\
\hline $1200-900$ & estiramento C-O-C & polissacarídeos & \\
\hline$\sim 700-400$ & - & $\begin{array}{c}\text { clorofilas, carotenoides, } \\
\text { outros pigmentos }\end{array}$ & (Golterman, 1975) \\
\hline
\end{tabular}

Fonte: O Autor.

Figura 4 - Comparação da mudança de intensidade da banda de estiramento C-O na região de $1300-1100 \mathrm{~cm}^{-1}$

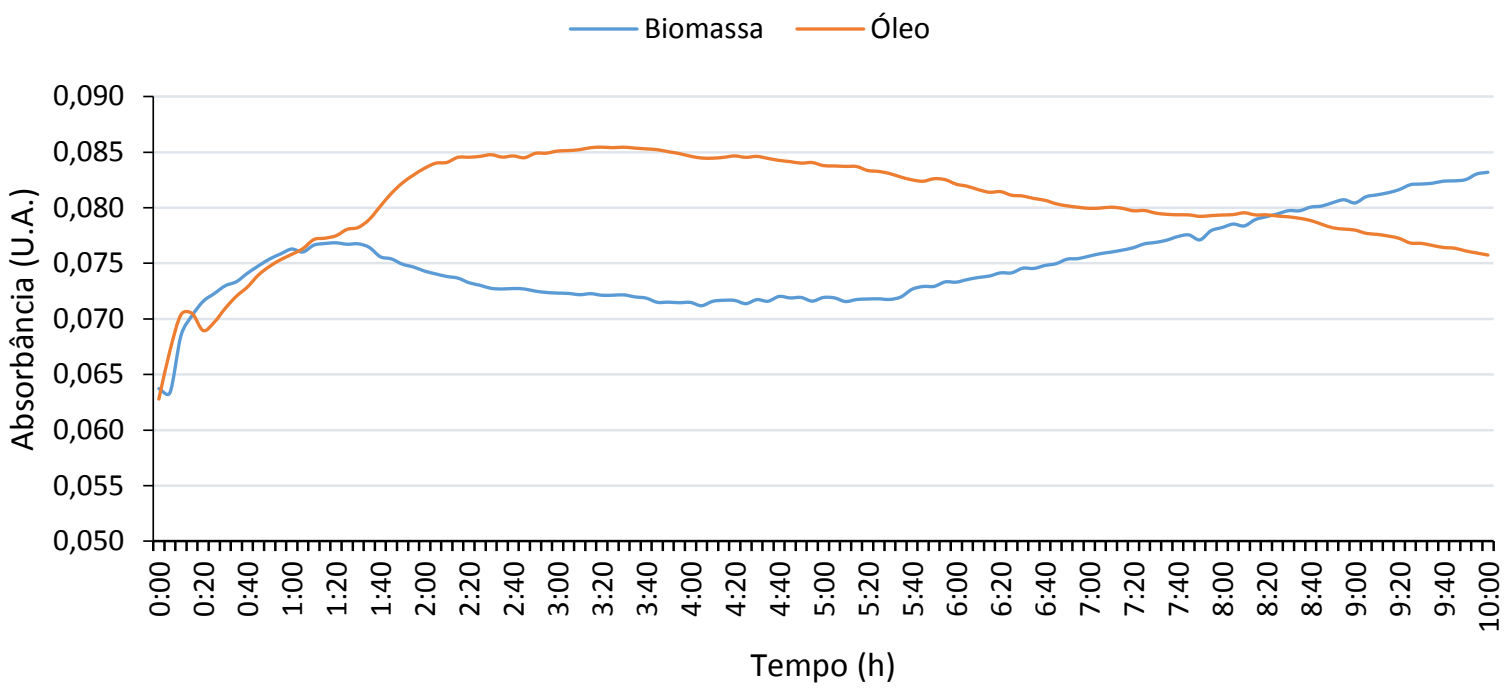

Fonte: O Autor.

Este resultado é importante para indicar que o conteúdo lipídico, ao ser extraído como TAG ou sendo extraído diretamente da célula com transformação in situ dos TAG em EMAG, apresenta comportamento similar com relação à reação propriamente dita. A diferença é que nos experimentos in situ, algumas modificações observadas nos espectros ao longo do tempo de 
observação, podem estar relacionadas à lise de outras moléculas no meio reacional, provenientes da fase não lipídica da biomassa.

\subsubsection{Cromatografia gasosa}

As análises cromatográficas dos ensaios realizados apresentam o perfil cromatográfico de EMAG característico. Desmodesmus sp. possui majoritariamente em sua composição lipídica, os ácidos palmítico (C16:0), oleico (C18:1) e linoleico (C18:2) e $\alpha$-linolênico (C18:3), podendo chegar até 18,73 \% do peso de sua biomassa seca na produção com suporte de $\mathrm{CO}_{2}$ (GRESSLER et al., 2014).

As reações de transesterificação apresentaram ésteres saturados, monoinsaturados e poliinsaturados. O grau de saturação dos EMAGs afeta diretamente a qualidade do biodiesel. Uma quantidade elevada de EMAGs poli-insaturados impacta positivamente nas propriedades de escoamento do biocombustível, especialmente em ambientes de baixa temperatura, mas pode afetar negativamente sua estabilidade oxidativa. EMAGs saturados possuem excelentes propriedades de combustão, porém podem causar problemas de fluxo em temperaturas baixas (D’ALESSANDRO e ANTONIOSI FILHO, 2016).

Os ensaios realizados exibiram altos teores de EMAG insaturados, como mostra a Tabela 4. Destaca-se o conteúdo de C18:1 (ácido oleico), o qual apresenta um balanço nas suas propriedades combustíveis, o que inclui ponto de entupimento de filtro a frio, calor de combustão, viscosidade e estabilidade oxidativa, que são determinadas pela sua estrutura (LEE et al., 2010).

A composição da biomassa varia muito com relação à espécie de microalga utilizada, e, portanto, o ácido oleico é um dos componentes que varia visivelmente influenciando diretamente na indicação da microalga para biodiesel. Navarro López et al. (2016) com a microalga Nannochloropsis gaditana como fonte lipídica e utilizando Novozym 435 como catalisador em uma reação in situ obtiveram 4,6 \% de C18:1, porém um valor superior de EMAGs de cadeias longas e poli-insaturados, como o C20:4 (7,3\%) e C20:5 (30,3). Com a microalga Desmodesmus brasiliensis, Nascimento et al. (2013) obtiveram 42,42 \% de ácido oleico extraído da biomassa. 
Tabela 4 - Proporção relativa (\%) a área total de ésteres identificados nos cromatogramas dos EMAGs encontrados por GC-DIC, para amostras dos dois métodos de transesterificação estudados.

\begin{tabular}{cccccc}
\hline & Óleo derivatizado & \multicolumn{2}{c}{ In situ } & \multicolumn{2}{c}{ Método tradicional } \\
\hline & & Média (\%) & Desvio padrão (\%) & Média (\%) & Desvio padrão (\%) \\
\cline { 2 - 5 } C10:0 & 0,00 & 2,30 & 1,10 & 0,24 & 0,34 \\
C12:0 & 0,00 & 1,36 & 0,97 & 1,94 & 1,38 \\
C14:0 & 1,67 & 1,94 & 1,41 & 6,17 & 1,16 \\
C14:1 & 0,00 & 2,19 & 1,61 & 0,69 & 0,97 \\
C16:0 & 4,72 & 2,78 & 0,44 & 3,70 & 1,69 \\
C16:1 & 4,72 & 1,10 & 0,78 & 2,55 & 2,40 \\
C18:0 & 0,00 & 1,28 & 1,81 & 6,37 & 3,39 \\
C18: $1^{\text {a }}$ & 0,28 & 2,64 & 3,73 & 2,45 & 1,77 \\
C18: $1^{\text {a }}$ & 0,00 & 0,84 & 1,19 & 1,89 & 2,67 \\
C18:1 & 2,06 & 11,42 & 4,75 & 5,16 & 4,80 \\
C18: ${ }^{\text {a }}$ & 0,00 & 2,64 & 3,73 & 0,00 & 0,00 \\
C18: & 2,40 & 5,36 & 4,59 & 6,34 & 5,53 \\
C18:3 & 38,24 & 5,12 & 1,14 & 13,48 & 9,33 \\
C20:0 & 38,24 & 0,00 & 0,00 & 0,00 & 0,00 \\
C20:1 & 7,19 & 2,44 & 3,45 & 2,82 & 3,99 \\
C20:4n-6 & 0,49 & 7,18 & 5,25 & 2,48 & 3,51 \\
C22:0 & 0,00 & 6,25 & 8,84 & 5,88 & 8,31 \\
C22:6n-3 & 0,00 & 1,42 & 2,01 & 0,00 & 0,00 \\
C24:0 & 0,00 & 41,72 & 11,11 & 37,35 & 13,47 \\
C24:1 & 0,00 & 0,00 & 0,00 & 0,50 & 0,70 \\
\hline
\end{tabular}

Fonte: O Autor.

Assim, com base na composição de ácidos graxos do biodiesel é possível prever as propriedades que atestam a qualidade do mesmo. Estas propriedades podem ser definidas pela estrutura molecular dos EMAGs e variam de acordo com o tamanho da cadeia e número ou posição de ligações duplas (NASCIMENTO et al., 2013). Dentre os parâmetros que possuem maior influência destacam-se o número de cetanos, índice de saponificação, índice de iodo, ponto de entupimento de filtro a frio e viscosidade entre todas as propriedades calculadas a partir da composição dos EMAGs, encontrados na Tabela 5.

O índice de iodo (II) mede o grau de instauração do combustível e aumenta proporcionalmente ao número de ligações duplas. Um alto II indica baixa estabilidade química, pois há um maior número de grupos reativos na molécula, aumentando a possibilidade de oxidação e formação de depósitos. Na Europa, os valores máximos estabelecidos para biodiesel são $120 \mathrm{~g} \mathrm{I}_{2} 100 \mathrm{~g}^{-1}$ (NASCIMENTO et al., 2013). Os ensaios que apresentaram valores baixos, 73,14 g I $100 \mathrm{~g}^{-1}$ in situ e 70,38 g I $100 \mathrm{~g}^{-1}$ a partir do óleo extraído são semelhantes ao de Song et al. (2013), que, utilizando a espécie Scenedesmus obliqnus, obtiveram II de 70,3 g I $100 \mathrm{~g}^{-1}$. 
O número de cetanos (NC) indica o tempo de atraso da ignição do combustível. Quanto maior seu valor, mais curto o tempo de ignição. Cadeias de ésteres metílicos muito longas aumentam a viscosidade e densidade do biodiesel, o que irá diminuir com o aumento de ligações duplas em sua estrutura. Os ensaios realizados superam os padrões europeus estabelecidos de NC mínimo de 51 (NASCIMENTO et al., 2013). Talebi et al. (2013), utilizando a cepa de Amphora sp., obtiveram biodiesel com NC de 62,33. O alto valor da estabilidade oxidativa do ensaio in situ comparado com o tradicional deve-se ao fato de que TAGs, ao serem extraídos, já começam a sofrer oxidação, o que não ocorre com os TAG no meio in situ, uma vez que a fração lipídica somente foi liberada durante a reação, possuindo uma degradação menor em contato com o oxigênio.

Os ésteres encontrados no biodiesel produzido a partir de Desmodesmus sp. possuem longas cadeias. Em relação ao PEFF, quanto maior a cadeia carbônica ou o grau de saturação do biodiesel, pior suas propriedades de temperatura, embora este parâmetro possa ser corrigido com o uso de aditivos (NASCIMENTO et al., 2013).

\section{Tabela 5 - Propriedades estimadas do biodiesel produzido nos diferentes ensaios}

\begin{tabular}{ccc}
\hline Propriedades & Transesterificação In situ & Transesterificação do óleo \\
\hline AGS & 57,63 & 61,63 \\
AGMI & 21,08 & 15,35 \\
AGPI & 19,08 & 22,3 \\
GI & 59,24 & 59,95 \\
VS & 176,51 & 182,92 \\
II & 73,14 & 70,38 \\
NC & 60,77 & 60,3 \\
FCSL & 93,73 & 87,04 \\
PEFF & 227,99 & 256,98 \\
PN & $-3,53$ & $-3,05$ \\
PAE & 67,14 & 56,57 \\
PBAE & 37,06 & 38,26 \\
EO & 13,84 & 8,54 \\
MVA & 39,34 & 39,83 \\
VC & 1,67 & 1,65 \\
$\rho$ & 0,85 & 0,86 \\
\hline
\end{tabular}

AGS = ácidos graxos saturados; AGMI = ácidos graxos monoinsaturados; AGPI = ácidos graxos poli-insaturados; GI = grau de instauração; VS = valor de saponificação (em mg de KOH necessários para saponificar $1 \mathrm{~g}$ de óleo); $I I$ = índice de iodo (em g $\left.I_{2} 100 \mathrm{~g}^{-1}\right) ; N C=$ número de cetanos; $F C L S=$ fator de cadeia saturada longa; PEFF = ponto de entupimento de filtro a frio $\left(\mathrm{em}^{\circ} \mathrm{C}\right) ; \mathrm{PN}=$ ponto nuvem $\left(\mathrm{em}{ }^{\circ} \mathrm{C}\right) ; \mathrm{PAE}=$ posição alílica equivalente; $P B A E$ = posição bis-alílica equivalente; $E O=$ estabilidade oxidativa (em horas); $M V A$ = maior valor de aquecimento; $V C$ = viscosidade cinemática $\left(\mathrm{em} \mathrm{mm^{2 }} \mathrm{s}^{-1}\right) ; \rho=$ densidade $\left(\mathrm{g} \mathrm{cm}^{-3}\right)$

Fonte: O Autor 
Um parâmetro que ficou abaixo do estabelecido por órgãos internacionais é a viscosidade cinemática, sendo que no padrão americano o biodiesel deve ser caracterizado com uma viscosidade entre 1,9 e $6,0 \mathrm{~mm}^{2} \mathrm{~s}^{-1}$ (KNOTHE e STEIDLEY, 2005). A viscosidade dos combustíveis afeta a ignição, a pressão de injeção/pulverização de combustível, influenciando o desempenho do motor, a combustão e emissões de escape.

\section{CONCLUSÃO}

O método de transesterificação enzimática in situ a partir de biomassa de Desmodesmus sp. em meio reacional com solvente orgânico foi possível. Apresenta como produto um biodiesel com perfil similar ao obtido pelo método enzimático tradicional partindo da fração lipídica extraída. O emprego do monitoramento da reação por infravermelho permitiu identificar que a reação alcançou o equilíbrio químico após duas horas e entender que a extração na forma de ésteres metílicos diretamente da célula da microalga, havia se esgotado. A extração dos lipídios empregando o método enzimático, conforme desenvolvido neste trabalho, pode levar a melhorias do processo com relação aos impactos ambientais e financeiros, uma vez que foi reduzida uma etapa. Estes impactos devem ser avaliados no futuro, bem como, comparar a transesterificação in situ enzimática com a química, buscando determinar o grau de conversão em relação ao tempo.

\section{AGRADECIMENTOS}

Ao CNPq pela bolsa de iniciação científica e pela bolsa produtividade (309652/2016-2), ao Ministério de Ciência, Tecnologia, Inovações e Comunicações (01.0144.00/2010). A Secretaria de Desenvolvimento Econômico, Ciência e Tecnologia do RS (209-2551/14-1) e ao TECNOUNISC, através do Centro de Excelência em Produtos e Processos Oleoquímicos e Biotecnológicos.

\section{REFERÊNCIAS}

AMARO, H. M., et al. Microalgae: an alternative as sustainable source of biofuels? Energy, v. 44, n. 1, p. 158-166, 2012. https://doi.org/10.1016/j.energy.2012.05.006

ASSOCIATION OF OFFICIAL ANALYTICAL CHEMISTS, A. Official methods of analysis. Vol. II, 1990. 
BEER, T. et al. The health impacts of ethanol blend petrol. Energies,v. 4, n. 2, p. 352-367, 2011. doi:10.3390/en4020352

BLIGH, E. G.; DYER, W. J. A rapid method of total lipid extraction and purification. Canadian journal of biochemistry and physiology, v. 37, n. 8, p. 911-917, 1959. DOI:10.1139/059-099

CAMPANELLA, L. et al. An algal biosensor for the monitoring of water toxicity in estuarine environments. Water Research, v. 35, n. 1, p. 69-76, 2001. https://doi.org/10.1016/S0043$\underline{1354(00) 00223-2}$

CHEN, C.-Y. et al. Microalgae-based carbohydrates for biofuel production. Biochemical Engineering Journal, v.78, p. 1-10, 2013. DOI: 10.1016/j.bej.2013.03.006

CHISTI, Y. Biodiesel from microalgae. Biotechnology advances, v. 25, n. 3, p. 294-306, 2007. https://doi.org/10.1016/j.biotechadv.2007.02.001

CHIU, S.-Y. et al. Reduction of CO2 by a high-density culture of Chlorella sp. in a semicontinuous photobioreactor. Bioresource Technology, v. 99, n. 9, p. 3389-3396, 2008. https://doi.org/10.1016/j.biortech.2007.08.013

D’AlESSANDRO, E. B.; ANTONIOSI FILHO, N. R. Concepts and studies on lipid and pigments of microalgae: A review. Renewable and Sustainable Energy Reviews, v. 58, p. 832841, 2016. https://doi.org/10.1016/j.rser.2015.12.162

DE OLIVEIRA RANGEL-YAGUI, C. et al. Chlorophyll production from Spirulina platensis: cultivation with urea addition by fed-batch process. Bioresource technology, v. 92, n. 2, p.133141, 2004. https://doi.org/10.1016/j.biortech.2003.09.002

DE SOUZA, A. V. A.; CAJAIBA DA SILVA, J. O. F., Biodiesel Synthesis Evaluated by Using Real-Time ATR-FTIR. Organic Process Research \& Development, v. 17, n. 1, p. 127-132, 2012. DOI: $10.1021 / \mathrm{op} 300318 \mathrm{k}$

DEL CAMPO, J. A. et al. Outdoor cultivation of microalgae for carotenoid production: current state and perspectives. Applied microbiology and biotechnology, v. 74, n. 6, p. 1163-1174, 2007. DOI: $10.1007 / \mathrm{s} 00253-007-0844-9$

DONG, T. et al. Two-step in situ biodiesel production from microalgae with high free fatty acid content. Bioresource technology, v. 136, p. 8-15, 2013. https://doi.org/10.1016/j.biortech.2013.02.105

GO, A. W. et al. Developments in in-situ (trans) esterification for biodiesel production: A critical review. Renewable and Sustainable Energy Reviews, v. 60, p. 284-305, 2016. https://doi.org/10.1016/j.rser.2016.01.070

GOLTERMAN, H. L. Algae and their Pigments. Developments in Water Science, v. 2, p. 233247, 1975. https://doi.org/10.1016/S0167-5648(08)71070-0

Revista Jovens Pesquisadores, Santa Cruz do Sul, v.7, n. 2, p. 26-45, jul./dez. 2017. 
GRESSLER, P. et al. Cultivation of Desmodesmus subspicatus in a tubular photobioreactor for bioremediation and microalgae oil production. Environmental technology, v. 35, n. 2, p. 209-219, 2014. DOI: $\underline{10.1080 / 09593330.2013 .822523}$

GULDHE, A. et al. Biodiesel synthesis from microalgae using immobilized Aspergillus niger whole cell lipase biocatalyst. Renewable Energy, v. 85, p. 1002-1010, 2016. https://doi.org/10.1016/j.renene.2015.07.059

HARUN, R., et al., Bioprocess engineering of microalgae to produce a variety of consumer products. Renewable and Sustainable Energy Reviews, v. 14, n. 3, p. 1037-1047, 2010. https://doi.org/10.1016/j.rser.2009.11.004

HODAIFA, G., et al., Use of industrial wastewater from olive-oil extraction for biomass production of Scenedesmus obliquus. Bioresource Technology, v. 99, n. 5, p. 1111-1117, 2008. https://doi.org/10.1016/j.biortech.2007.02.020

HOEKMAN, S. K., et al., Review of biodiesel composition, properties, and specifications. Renewable and Sustainable Energy Reviews, v. 16, n. 1, p. 143-169, 2012. https://doi.org/10.1016/j.rser.2011.07.143

HU, C.-W., et al., Pigment production by a new thermotolerant microalga Coelastrella sp. F50. Food chemistry, v. 138, n. 4, p. 2071-2078, 2013. DOI:10.1016/j.foodchem.2012.11.133

JUNEJA, A., et al., Effects of environmental factors and nutrient availability on the biochemical composition of algae for biofuels production: a review. Energies, v. 6, n. 9, p. 4607-4638, 2013. doi: $\underline{10.3390 / e n 6094607}$

KEERA, S. T., et al., Transesterification of vegetable oil to biodiesel fuel using alkaline catalyst. Fuel, v. 90, n. 1, p. 42-47, 2011. https://doi.org/10.1016/j.fuel.2010.07.046

KIM, S. e KIM, C. H., Bioethanol production using the sequential acid/alkali-pretreated empty palm fruit bunch fiber. Renewable Energy, v. 54, p. 150-155, 2013. https://doi.org/10.1016/j.renene.2012.08.032

KNOTHE, G.; STEIDLEY, K. R., Kinematic viscosity of biodiesel fuel components and related compounds. Influence of compound structure and comparison to petrodiesel fuel components. Fuel, v. 84, n. 9, p. 1059-1065, 2005. https://doi.org/10.1016/j.fuel.2005.01.016

LEE, J.-Y., et al., Comparison of several methods for effective lipid extraction from microalgae. $\begin{array}{lllllll}\text { Bioresource Technology, } & \text { v. } 101, \quad \text { n. } & 1, & \text { p. } & \text { S75-S77, } & 2010 .\end{array}$ https://doi.org/10.1016/j.biortech.2009.03.058

LI, Z., et al., Analysis of fatty acids in mouse cells using reversed-phase high-performance liquid chromatography. Chromatographia, v. 54, n. 7, p. 463-467, 2001. https://doi.org/10.1007/BF02491200 
LICHTENTHALER, H. K., Chlorophylls and carotenoids: pigments of photosynthetic biomembranes. Methods in enzymology, v. 148, n. 350-382, 1987. https://doi.org/10.1016/0076$\underline{6879(87) 48036-1}$

MALLICK, N., Biotechnological potential of immobilized algae for wastewater N, P and metal removal: a review. Biometals, $\quad$ v. 15, n. 4, p. 377-390, 2002. https://doi.org/10.1023/A:1020238520948

MAYERS, J. J., et al., Rapid determination of bulk microalgal biochemical composition by Fourier-Transform Infrared spectroscopy. Bioresource technology, v. 148, p. 215-220, 2013. https://doi.org/10.1016/j.biortech.2013.08.133

MUELLER, D., et al., Fourier transform infrared spectroscopy (FTIR) and multivariate analysis for identification of different vegetable oils used in biodiesel production. Sensors, v. 13, n. 4, p. 4258-4271, 2013. doi: 10.3390/s130404258

NASCIMENTO, I. A., et al., Screening Microalgae Strains for Biodiesel Production: Lipid Productivity and Estimation of Fuel Quality Based on Fatty Acids Profiles as Selective Criteria. BioEnergy Research, v. 6, n. 1, p. 1-13, 2013. https://doi.org/10.1007/s12155-012-9222-2

NAVARRO LÓPEZ, E., et al., Fatty acid methyl ester production from wet microalgal biomass by lipase-catalyzed direct transesterification. Biomass and Bioenergy, v. 93, p. 6-12, 2016. https://doi.org/10.1016/j.biombioe.2016.06.018

OLIVEIRA, A. D. D., et al., Comprehensive near infrared study of Jatropha oil esterification with ethanol for biodiesel production. Spectrochimica Acta Part A: Molecular and Biomolecular Spectroscopy, v. 170, p. 56-64, 2017. doi: 10.1016/j.saa.2016.07.006.

SAMORÌ, G., et al., Growth and nitrogen removal capacity of Desmodesmus communis and of a natural microalgae consortium in a batch culture system in view of urban wastewater treatment: Part I. Water Research, v. 47, n. 2, p. 791-801, 2013. doi: 10.1016/j.watres.2012.11.006

SINGH, B., et al., Towards a sustainable approach for development of biodiesel from plant and microalgae. Renewable and sustainable Energy reviews, v. 29, p. 216-245, 2014. https://doi.org/10.1016/j.rser.2013.08.067

SONG, M., et al., Evaluation of the potential of 10 microalgal strains for biodiesel production. $\begin{array}{llllll}\text { Bioresource Technology, } & \text { v. } & \text { 141, } & \text { p. } & \text { 245-251, } & \end{array}$ https://doi.org/10.1016/j.biortech.2013.02.024

SOUSA, F. P., et al., Thermogravimetry and Viscometry for Assessing the Ester Content (FAME and FAEE). Fuel Processing Technology, v. 109, p. 133-140, 2013. https://doi.org/10.1016/j.fuproc.2012.09.049 
TALEBI, A. F., et al., Fatty acids profiling: a selective criterion for screening microalgae strains for biodiesel production. Algal Research, v. 2, n. 3, p. 258-267, 2013. https://doi.org/10.1016/j.algal.2013.04.003

VELASQUEZ-ORTA, S. et al. Alkaline in situ transesterification of Chlorella vulgaris. Fuel, v. 94, p. 544-550, 2012. https://doi.org/10.1016/j.fuel.2011.11.045

VIJAYARAGHAVAN, K.; HEMANATHAN, K. Biodiesel production from freshwater algae. Energy \& Fuels, v. 23, n. 1, p. 5448-5453, 2009. DOI: 10.1021/ef9006033

ZHANG, W.-B., Review on analysis of biodiesel with infrared spectroscopy. Renewable and Sustainable Energy Reviews, v. 16, n. 8, p. 6048-6058, 2012. https://doi.org/10.1016/j.rser.2012.07.003

ZMORA, O.; RICHMOND, A. Microalgae for Aquaculture. Handbook of Microalgal Culture: Biotechnology and Applied Phycology, 2004. DOI: 10.1002/9780470995280.ch20 